\title{
Containing COVID-19 in a specialised neurology centre: the risks of presymptomatic transmission
}

\author{
Liang En lan Wee ${ }^{1,2}$ (1) Siew Yee Thien ${ }^{2} \cdot$ Shekhawat Ravindra Singh ${ }^{3} \cdot$ Moi Lin Ling ${ }^{4} \cdot$ Indumathi Venkatachalam ${ }^{2,4}$
}

Received: 15 June 2020 / Accepted: 21 June 2020 / Published online: 27 June 2020

(C) Fondazione Società Italiana di Neurologia 2020

\section{To the Editor:}

We read with interest the article by Baracchini et al., describing an integrated care pathway for acute stroke during a COVID-19 pandemic, in which patients with respiratory symptoms or fever were managed with appropriate precautions as suspected COVID-19 patients [1]. Indeed, given the growing literature on neurological manifestations associated with SARS-CoV-2 [2], there is consensus that care pathways for neurology patients, including acute stroke care, need to be modified to minimise potential exposure and incorporate SARS-CoV-2 screening in areas with high prevalence [3]. Various strategies have been proposed, including universal screening for all stroke patients and utilising pulmonary imaging to prioritise patients for screening [4]. However, given that a minority may be asymptomatic [5] and pulmonary imaging alone may be insufficient as a triage strategy, cases may still be missed. We would like to highlight the risks posed by pre-symptomatic transmission.

In Singapore, community transmission of COVID-19 was reported since early-February 2020. The National Neuroscience Institute (NNI) is the largest specialised neurological centre in Singapore; its Outram campus is co-located with the Singapore General Hospital (SGH), the largest tertiary hospital in

Re: Baracchini, C., Pieroni, A., Viaro, F. et al. Acute stroke management pathway during Coronavirus-19 pandemic. Neurol Sci 41, 1003-1005 (2020).

Liang En Ian Wee

ian.wee@mohh.com.sg

1 Singhealth Infectious Diseases Residency, Singapore, Singapore

2 Department of Infectious Diseases, Singapore General Hospital, Singapore, Singapore

3 Department of Neurology, National Neuroscience Institute, Singapore, Singapore

4 Department of Infection Prevention and Epidemiology, Singapore General Hospital, Singapore, Singapore
Singapore. During the COVID-19 outbreak, all neurology inpatients admitted to the Outram campus of the NNI were screened using a risk-stratified approach. If patients had suspicious travel/ epidemiology history, respiratory symptoms, or pulmonary infiltrates on imaging, they were placed in a dedicated ward where SARS-CoV-2 and other common respiratory viruses were tested for and HCWs used N95 masks, disposable gowns, gloves and goggles, until COVID-19 was excluded. Positive patients were transferred to isolation wards, while negatives were decanted to the general ward. If acute interventions (e.g. thrombolysis or thrombectomy) were necessary prior to exclusion of COVID19 , patients were managed as if they were positive.

Over a 16-week period (5 February-30 May 2020), 198 neurology patients were tested for COVID-19 using a risk-stratified approach. The majority were tested due to pulmonary infiltrates on baseline imaging $(62.6 \%, 124 / 198)$; $47.4 \%$ (94/198) had concomitant respiratory symptoms; and 20.8\% (41/198) were tested due to suspicious epidemiology. Around 20.7\% (41/198) required stroke unit/intensive care. A total of 8 neurology inpatients $(4.0 \%, 8 / 198)$ tested positive for respiratory viruses (2 SARSCoV-2, 6 other respiratory viruses), compared with $14.7 \%$ (951/ 6465) of non-neurology medical inpatients in our co-located general hospital testing positive over the same period (606 SARS-CoV-2, 345 other respiratory viruses). The yield of respiratory virus testing was lower in neurology inpatients (odds ratio, $\mathrm{OR}=0.24,95 \% \mathrm{CI}=0.12-0.50, p<0.001)$.

Details of the two neurology inpatients with COVID-19 are found in Fig. 1. Of note, both had no respiratory symptoms or pulmonary infiltrates; acute infarct was confirmed on neuroimaging. Due to high-risk epidemiology, both patients were managed in isolation areas with full precautions prior to confirmation of COVID-19, mitigating onward transmission. Although a total of $63 \mathrm{HCWs}$ and 2 other patients came into contact with the index cases prior to the diagnosis of COVID-19, because of our risk-stratified approach to testing, healthcare-associated exposure was contained. Vigilance should be maintained for highrisk epidemiology, given the possibility of pre-symptomatic transmission. 
Fig. 1 Clinical details, imaging findings, and epidemiology investigations for neurology inpatients with acute stroke and concurrent COVID-19 infection at a specialised neurology centre in Singapore, during a COVID-19 outbreak $(N=2)$

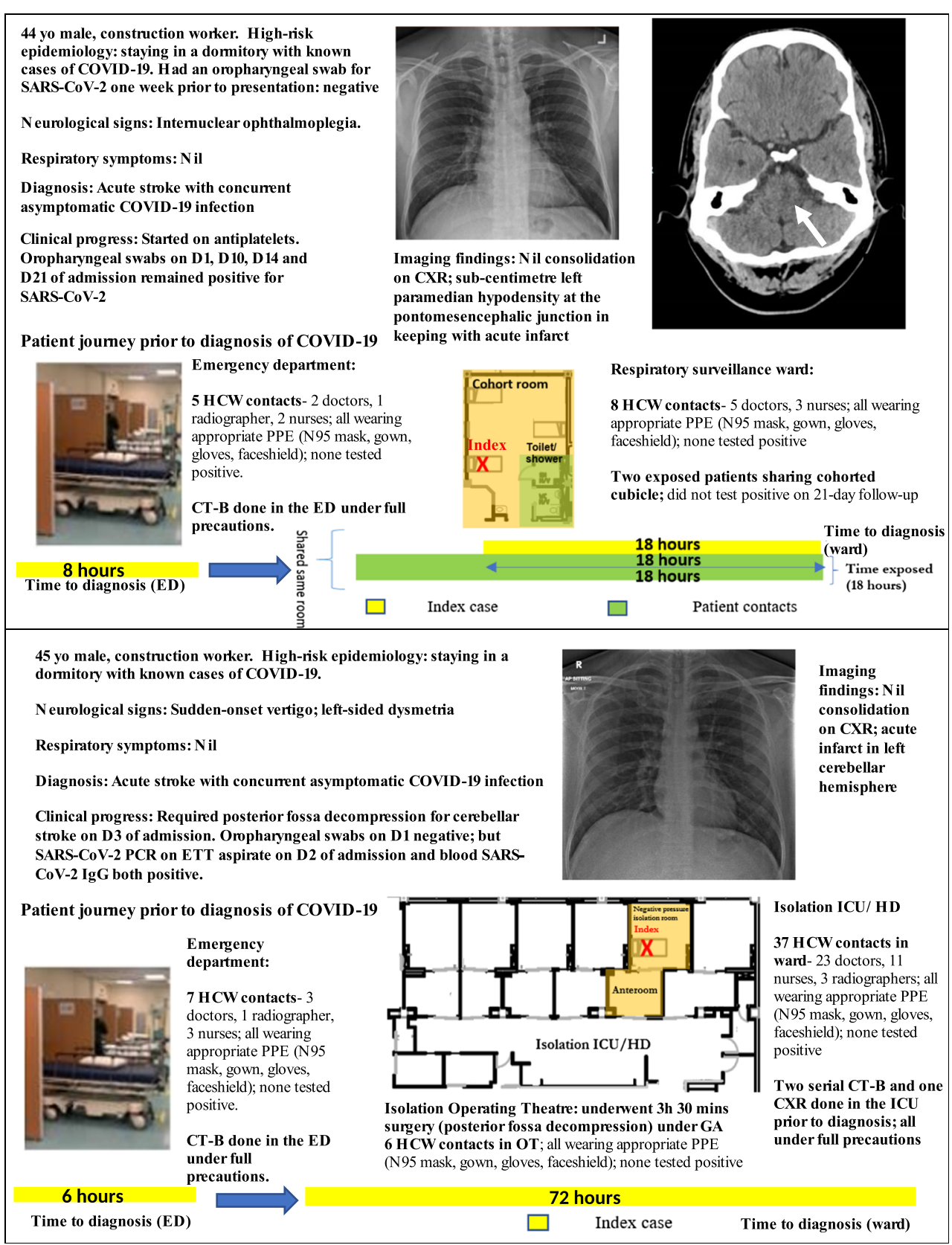

\section{Compliance with ethical standards}

Conflict of interest The authors declare that they have no conflicts of interest.

\section{References}

1. Baracchini C, Pieroni A, Viaro F, Cianci V, Cattelan AM, Tiberio I, Munari M, Causin F (2020) Acute stroke management pathway during Coronavirus-19 pandemic. Neurol Sci 41:1003-1005

2. Benussi A, Pilotto A, Premi E, Libri I, Giunta M, Agosti C, Alberici A, Baldelli E, Benini M, Bonacina S, Brambilla L, Caratozzolo S, Cortinovis M, Costa A, Piccinelli SC, Cottini E, Cristillo V, Delrio I, Filosto M, Gamba M, Gazzina S, Gilberti N, Gipponi S, Imarisio A,
Invernizzi P, Leggio U, Leonardi M, Liberini P, Locatelli M, Masciocchi S, Poli L, Rao R, Risi B, Rozzini L, Scalvini A, Schiano di Cola F, Spezi R, Vergani V, Volonghi I, Zoppi N, Borroni B, Magoni M, Pezzini A, Padovani A (2020) Clinical characteristics and outcomes of inpatients with neurologic disease and COVID-19 in Brescia, Lombardy, Italy. Neurology:10.1212/ WNL.0000000000009848. https://doi.org/10.1212/WNL. 0000000000009848

3. Ford T, Curiale G, Nguyen TN, Aparicio H, Hamlyn EK, Gangadhara S, Cervantes-Arslanian AM, Greer D, Romero JR, Shulman JG (2020) Optimization of resources and modifications in acute ischemic stroke. Care in response to the global COVID-19 pandemic. J Stroke Cerebrovasc Dis. https://doi.org/10.1016/j. jstrokecerebrovasdis.2020.104980

4. Kwee RM, Krdzalic J, Fasen BACM, de Jaegere TMH (2020) COVID-19 CT Investigators South-East Netherlands (CISEN) Study Group. CT scanning in suspected stroke or head trauma: is it 
worth to go the extra mile and include the chest to screen for COVID-19 infection? AJNR Am J Neuroradiol. https://doi.org/10. 3174/ajnr.A6607

5. Wei WE, Li Z, Chiew CJ, Yong SE, Toh MP, Lee VJ (2020) Presymptomatic transmission of SARS-CoV-2 - Singapore,
January 23-March 16, 2020. MMWR Morb Mortal Wkly Rep 69(14):411-415

Publisher's note Springer Nature remains neutral with regard to jurisdictional claims in published maps and institutional affiliations. 